\title{
Bioanalytical Application of SERS Immunoassay for Detection of Prostate-Specific Antigen
}

\author{
Kyung Jin Yoon, ${ }^{a}$ Hyeong Kuyn Seo, ${ }^{a}$ Hoon Hwang, Dongjin Pyo, In-Yong Eom, ${ }^{\dagger}$ Jong Hoon Hahn, ${ }^{\star}$ and Young Mee Jung ${ }^{*}$ \\ Department of Chemistry, Institute for Molecular Science and Fusion Technology, Kangwon National University, \\ Chunchon 200-701, Korea. ${ }^{*}$ E-mail: ymjung@kangwon.ac.kr \\ ${ }^{\dagger}$ Department of Life Chemistry, Catholic University of Daegu, Gyeongsan 712-702, Korea \\ ${ }^{\ddagger}$ Department of Chemistry, BK School of Molecular Science, POSTECH, Pohang 790-784, Korea \\ Received February 18, 2010, Accepted March 2, 2010
}

\begin{abstract}
We demonstrate the possible application of the sandwich type surface-enhanced Raman scattering (SERS) immunoassay using antigen-antibody binding for detection of prostate-specific antigen (PSA) in cancer cells. In this sandwich type of SERS immunoassay, to capture antigens onto the immobilized layer of antibodies on the gold substrate we prepared the monolayer of gold nanoparticles on the APTMS-derivatized surface of a glass slide by using the SAM technique. This sandwich type of SERS immunoassay in which antigens on the substrate specifically capture antibodies on a Raman reporter (DSNB coated gold nanoparticles with R6G) could successfully detect PSA at low levels. A strong SERS spectrum of Raman reporter was observed only with a substrate in which PSA is present.
\end{abstract}

Key Words: Surface-enhanced Raman scattering (SERS), Immunoassay, Prostate-specific antigen (PSA), Raman reporter, Gold nanopaticles

\section{Introduction}

Surface-enhanced Raman scattering (SERS) spectroscopy has recently received much attention due to its considerable potential in highly selective and sensitive detection of molecules of interest even in single-molecule detection. ${ }^{1-30}$ SERS in which the scattering cross-sections are dramatically enhanced for molecules adsorbed on metallic nanostructure has been a promising candidate for various applications in nanoscience and nanotechnology. Particular interest has thus been focused on applications of molecular sensors operating via SERS for biochemical studies, clinical diagnoses, and environmental monitoring. ${ }^{28}$ Especially, SERS immunoassays based on antigen-antibody binding have been reported for biomarkers for early cancer diagnosis. ${ }^{12-18,21-24,26,30}$ For successful application of SERS immunoassays for biological sensors, people usually used novel metal nanoparticles modified to integrate bioselective species (e.g., antibodies) with molecular labels for the generation of intense, biolyte-selective SERS responses in immunoassays and other bioanalytical applications.

Immunoassays, with high selectivity and affinity of antibody molecules to their corresponding antigens, have been widely exploited for analytical purposes in the field of clinical diagnoses. ${ }^{31-36}$ Immunoassay readout methods have been based on fluorescence, electrochemistry, chemiluminescence, Rayleigh scattering, and Raman scattering. Fluorescence spectroscopy has been one of the most widely used readout methods due to its high sensitivity. Raman spectroscopy has inherent problems such as extremely low sensitivity as an immunoassay readout method. However, immunoassay based on SERS provides a high sensitivity which is comparable to that of fluorescence spectroscopy.

Prostate-specific antigen (PSA), which is a glycoprotein con-

\footnotetext{
${ }^{\mathrm{a}}$ The authors contributed equally to this work.
}

sisting of one polypeptide chain, has been used as a serologic marker for detecting prostate cancer. Some types of immunoassay for PSA have been reported, such as fluorescence microscopy, surface plasmon resonance technology, lateral flow immunoassay, enzyme-linked immunosorbent assay (ELISA), radioimmunoassay, immunochromatographic assay system, and SERS based on immunoassays. ${ }^{15-17,26,37-44}$ In this study, we applied a sandwich type SERS immunoassay for the detection of PSA with high sensitivity. It shows the possible application of SERS immunoassay with monoclonal antibodies that is sensitive enough for analysis of PSA in human blood samples or cancer cells.

\section{Experimental Section}

Prostate-specific monoclonal antibody (anti-PSA-mAb) was obtained from Boditech Co. (Korea). Prostate-specific antigen (PSA), gold (III) chloride hydrate $\left(\mathrm{HAuCl}_{4}, 99 \%\right)$, sodium citrate tribasic dehydrate $\left(\mathrm{Na}_{3}\right.$ citrate, $99 \%$ ), and 3-aminopropyl trimethoxysilane (APTMS, $\mathrm{H}_{2} \mathrm{~N}\left(\mathrm{CH}_{2}\right)_{3} \mathrm{Si}\left(\mathrm{OCH}_{3}\right)_{3}, 97 \%$ ) were purchased from Sigma Aldrich at highest purity available and used as received without further purification. N-hydroxysuccinimide (NHS), 1,3-dicyclohexylcarbodiimide (DCCD), and 5,5'dithiobis(2-nitrobenzoic acid) (DNBA) were purchased from Alfa Aesar at the highest purity available and used as received without further purification.

Gold colloidal nanoparticles were prepared by the aqueous reduction of $\mathrm{HAuCl}_{4}$ solution $\left(100 \mathrm{~mL}, 10^{-2} \%\right)$ with trisodium citrate solution (10 $\mathrm{mL}$ of $1 \%$ ) using a process developed by Frens. ${ }^{45}$ The particle size of the gold colloidal nanoparticles can be controlled by adjusting the amount of dropping solution. The gold colloidal nanoparticle solution showed an absorption maximum near $523 \mathrm{~nm}$. The size of the gold colloidal nanoparticles was 13 - $20 \mathrm{~nm}$ from the high-resolution transmission electron microscopy (TEM) images. Figure 1 shows a TEM image of 


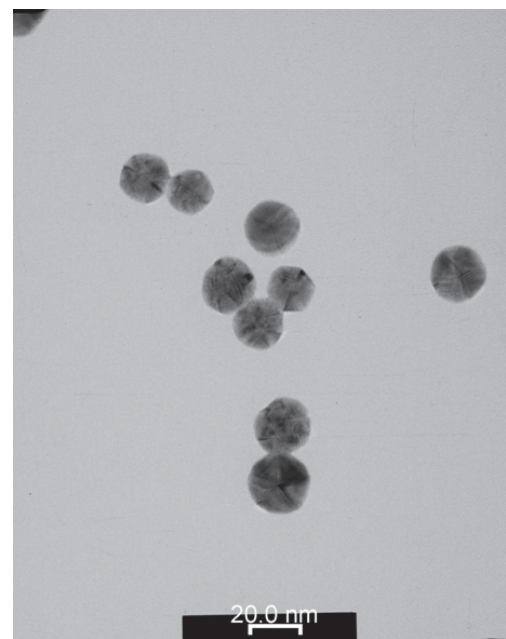

Figure 1. TEM image of colloidal gold nanoparticles.

colloidal gold nanoparticles.

The high-resolution TEM images were obtained with a JEOL JEM 2010 transmission electron microscope operated at $200 \mathrm{kV}$. Field emission scanning electron microscope (FE-SEM) images were obtained with a Hitachi S-4300 field emission scanning electron microscope operated at $15 \mathrm{kV}$. UV-vis absorption spectra were obtained with a Sinco S-3100 UV-vis spectrometer.

SERS spectra were recorded using a Jobin Yvon/HORIBA LabRam ARAMIS Raman spectrometer equipped with an integral BX 41 confocal microscope. The radiation from an air cooled HeNe laser (632.8 nm) was used as an excitation source. Raman scattering was detected with $180^{\circ}$ geometry using a multi channel air-cooled $\left(-70^{\circ} \mathrm{C}\right)$ charge-coupled device $(\mathrm{CCD})$ camera $(1024 \times 256$ pixels $)$.

For the SERS immunoassay, rhodamine $6 \mathrm{G}$ (R6G) adsorbed on colloidal gold nanoparticles was used as a Raman reporter in this study. R6G is a well-known SERS dye, which has been used in single-molecule detection. 5,5'-dithiobis (succinimidyl2-nitrobenzoate) (DSNB) was used for antibody conjugation on to gold nanoparticles. DSNB was synthesized and DSNBcoated Raman reporter was prepared by the method reported earlier. ${ }^{15,24}$ Antibody was coupled to R6G adsorbed on gold nanoparticles via the succinimidyl terminus of the DSNB-derived coating.

To prepare to capture antibody substrates, a 3-aminopropyl trimethoxysilane (APTMS)-derivatized surface of a glass slide was prepared by using the self-assembled monolayer (SAM) technique. SAM of APTMS was fabricated by immersing an acid-cleaning of the glass slide substrate for 24 hours, and then a successive gold nanoparticle layer was prepared by immersing the APTMS-derivatized substrate in a colloidal gold nanoparitcles solution for 24 hours.

Antibody was also adsorbed on the gold nanoparticle SAM surface via the succinimidyl terminus of the DSNB-derived coating. The PSA was conjugated onto the immobilized layer of antibody on the gold nanoparticle SAM substrate by immerging antibody-derivatized substrate in PSA solution (in Tris buffer) for 1 hour.

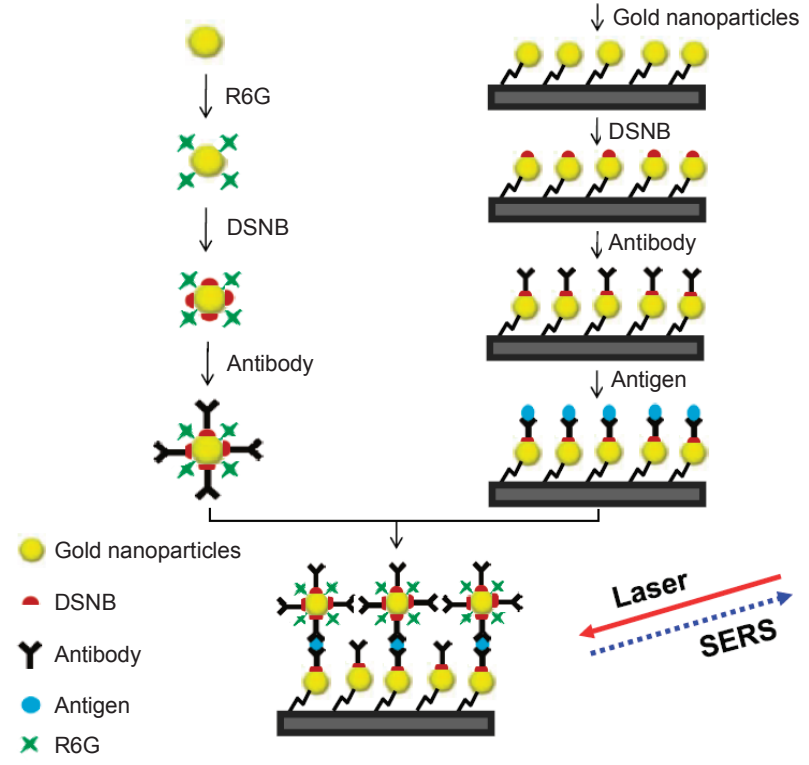

Figure 2. Scheme of the sandwich type SERS immunoassay process.

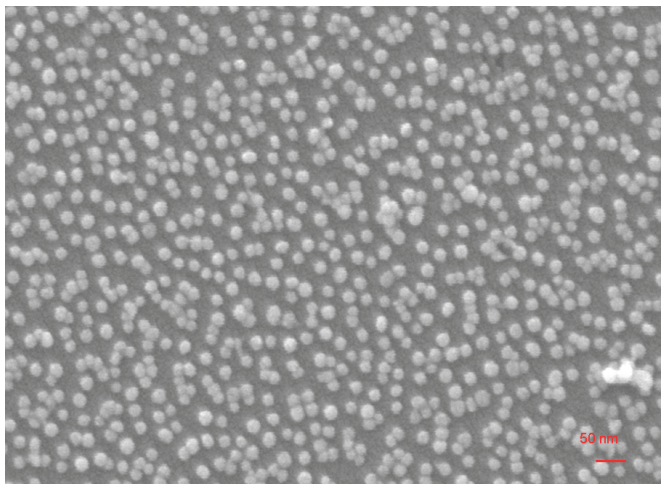

Figure 3. FE-SEM image of the gold nanoparticle monolayer on the APTMS-derivatized SAM substrate.

\section{Results and Discussion}

To demonstrate the possible application of SERS immunoassay for detection of antigen (PSA) in cancer cells, we performed the sandwich type SERS immunoassay process. Figure 2 depicts the scheme of the sandwich type SERS immunoassay process used in this study. As shown in Figure 2, this sandwich type of SERS immunoassay involves the immobilization of captured antibodies on a Raman reporter (DSNB coated gold nanoparticles with R6G), the conjugation of antigens onto the immobilized layer of antibodies on the gold nanoparticle SAM substrate, the immobilized antibodies capturing antigens on the surface, and SERS detection of the Raman reporter on which antigen is selectively conjugated with antibody.

Figure 3 shows a FE-SEM image of the gold nanoparticles monolayer on the APTMS-derivatized SAM substrate. It can be seen that the gold nanoparticles were homogeneously assembled on the APTMS-derivatized SAM substrate. This gold nano- 


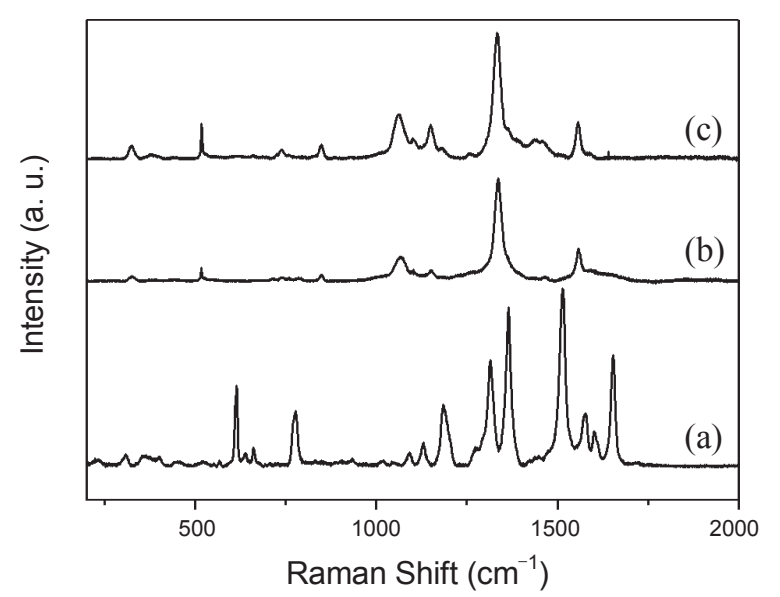

Figure 4. SERS spectra of (a) R6G $\left(10^{-6} \mathrm{M}\right)$ on colloidal gold nanoparticle, (b) the antibody $\left(2 \times 10^{-3} \mathrm{M}\right)$ conjugated onto the DSNB coated gold nanoparticles, and (c) PSA with antibody $\left(2 \times 10^{-3} \mathrm{M}\right)$ coupled to DSNB coated gold nanoparticles.

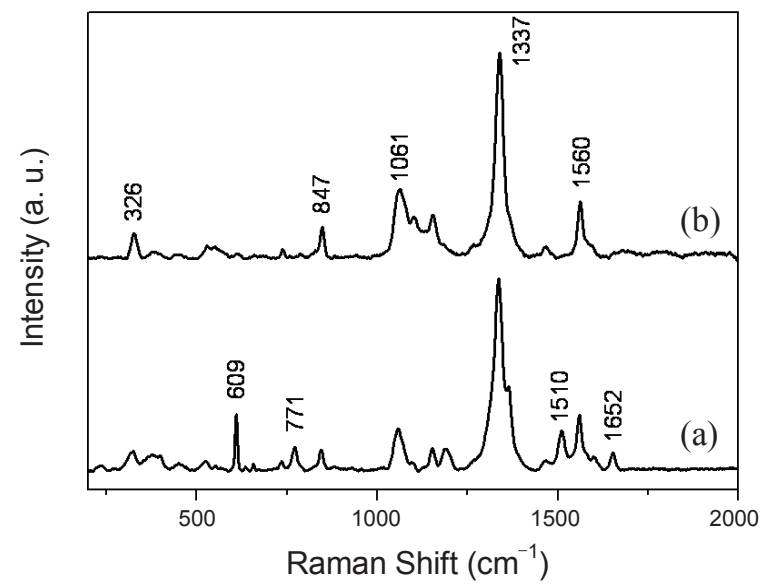

Figure 5. SERS spectra of Raman reporters of antibody on the substrate in which (a) PSA is present and (b) PSA is not present.

particles monolayer is a good substrate to capture antibody in this sandwich type of SERS immunoassay. By the specific antigen-antibody interaction, the PSA was thus conjugated successfully with antibody on the gold nanoparticles monolayer via the succinimidyl terminus of the DSNB.

Figure 4 shows the SERS spectra of R6G $\left(10^{-6} \mathrm{M}\right)$ on colloidal gold nanoparticles, the antibody $\left(2 \times 10^{-3} \mathrm{M}\right)$ conjugated onto colloidal gold nanoparticles via the succinimidyl terminus of the DSNB-derived coating, and the captured antigen (PSA) with antibody $\left(2 \times 10^{-3} \mathrm{M}\right)$ coupled to colloidal gold nanoparticles via the succinimidyl terminus of the DSNB-derived coating. As shown in Figure 4, the SERS spectrum of R6G adsorbed on colloidal gold nanoparticles is completely different from those of antibody and PSA coupled with antibody. The intensities of the Raman bands for R6G, even at very low concentration, are much stronger than those for the others. It means that R6G could be a good Raman reporter for detection of PSA by SERS immunoassay. In this study, R6G was thus used as a Raman reporter due to the low level of detection sensitivity, although the strongest Raman band at of DSNB on gold nanoparticles has been used as a Raman reporter for SERS immunoassay.

As mentioned above, the SERS immunoassay using antigenantibody binding is a powerful method for the detection of specific cancer detection. Figure 5(a) displays the SERS spectrum of the immobilized Raman reporter of antibody on the substrate of PSA captured with the immobilized antibody on the gold nanoparticle SAM surface. The immobilized Raman reporter of the antibody specifically extracts the available PSA when PSA is present on a substrate that is an immobilized layer of antibody on the gold naoparticle SAM surface. The SERS spectrum of the Raman reporter of antibody on the substrate of the immobilized antibody on a gold nanoparticle SAM surface in which PSA is not present is presented in Figure 5(b) for comparison. The bands at $1652,1510,771$, and $609 \mathrm{~cm}^{-1}$ assigned to the characteristic Raman bands for R6G are observed only for the substrate in which PSA is present as shown in Figure 5(a). It means that antibodies on the Raman reporter are successfully and selectively conjugated with antigen (PSA) on the immobilized antibody on the gold naoparticle SAM surface. We could finally detect PSA at very low levels $(\sim 1 \mathrm{pg} / \mathrm{mL})$ by using this SERS immunoassay method, which is within PSA concentration in human blood samples. Therefore, this SERS immunoassay method can be applied for sensitive detection of PSA in cancer cells.

\section{Conclusion}

An effective detection method based on SERS immunoassay using antigen-antibody binding has been introduced. For the possible application of SERS immunoassay for detection of antigen (PSA) in cancer cells, we performed the sandwich type SERS immunoassay process. Very interestingly, to capture antigen onto the immobilized layer of antibodies on the gold substrate, we prepared a monolayer of gold nanoparticles on the APTMS-derivatized surface of a glass slide by using the SAM technique. This sandwich type SERS immunoassay involves the immobilization of capture antibodies on a Raman reporter (DSNB coated gold nanoparticles with R6G), the conjugation of antigens onto the immobilized layer of antibodies on the gold nanoparticle SAM substrate, the immobilized antibodies capturing the antigens on the surface, and SERS detection of the Raman reporter on which antigen is selectively conjugated with antibody.

We demonstrate the potential of this sandwich type of SERS immunoassay to detect PSA successfully at very low levels. This powerful SERS immunoassay method would open a way for detection of PSA in human blood samples or cancer cells.

Acknowledgments. This work was supported by the National Research Foundation of Korea (NRF) grants funded by the Korea government (MEST) (No. 2009-0083707 and No. 20090087013) and the BK 21 program from the Ministry of Education, Science and Technology of Korea. The authors thank the Central Laboratory of Kangwon National University for the measurements of Raman spectra. 


\section{References}

1. Kneipp, K., Moskovits, M., Kneipp, H., Eds.; Surface-Enhanced Raman Scattering-Physics and Applications; Springer: Heidelberg and Berlin, 2006.

2. Aroca, R. Surface-Enhanced Vibrational Spectroscopy; John Wiley and Sons: Chichester, UK, 2006.

3. Schatz G. C.; Van Duyne, P. R. Handbook of Vibrational Spectroscopy Vol. 1: Electromagnetic Mechanism of Surface-Enhanced Spectroscopy; Chalmers, J. M., Griffiths, P. R., Eds.; John Wiley and Sons: Chichester, UK, 2002.

4. Chang R. K., Furtak, T. E., Eds.; Surface-Enhanced Raman Scattering; Plenum Press: New York, 1982.

5. Qian, X.-M.; Nie, S. M. Chem. Soc. Rev. 2008, 37, 912.

6. Graham, D.; Goodacre, R. Chem. Soc. Rev. 2008, 37, 883.

7. Kneipp, K.; Kneipp, H.; Itzkan, I.; Dasari, R. R.; Feld, M. S. Chem. Rev. 1999, 99, 2957.

8. Kneipp, K.; Kneipp, H.; Kartha, V. B.; Manoharan, R.; Deinum, G.; Itzkan, I.; Dasari, R. R.; Feld, M. S. Phys. Rev. E 1998, 57, R6281.

9. Kneipp, K.; Wang, Y.; Kneipp, H.; Perelman, L. T.; Itzkan, I.; Dasari, R. R.; Feld, M. S. Phys. Rev. Lett. 1997, 78, 1667.

10. Moskovits, M. Rev. Mod. Phys. 1985, 57, 783.

11. Kim, K.; Lee, Y. M.; Lee, H. B.; Shin, K. S. Appl. Mater. Interfaces 2009, 1, 2174.

12. Woo, M.-A.; Lee, S.-M.; Kim, G.; Baek, J.; Noh, M. S.; Kim, J. E.; Park, S. j.; Minai-Tehrani, A.; Park, S.-C.; Seo, Y. T.; Kim, Y.-K.; Lee, Y.-S.; Jeong, D. H.; Cho, M.-H. Anal. Chem. 2009, 81, 1008.

13. Chon, H.; Lee, S.; Son, S. W.; Oh, C. H.; Choo, J. Anal. Chem. 2009, 81, 3029.

14. Bao, F.; Yao, J.-L.; Gu, R.-A. Langmuir 2009, 25, 10782.

15. Wang, G.; Park, H.-Y.; Lipert, R. J. Anal. Chem. 2009, 81, 9643.

16. Stevenson, R.; Ingram, A.; Leung, H.; McMillan, D. C.; Graham, D. Analyst 2009, 134, 842.

17. Jehn, C.; Küstner, B.; Adam, P.; Marx, A.; Ströbel, P.; Schmuck, C.; Schlücker, S. Phys. Chem. Chem. Phys. 2009, 11, 7499.

18. Han, X. X.; Cai, L. j.; Guo, J.; Wang, C. X.; Ruan, W. D.; Han, W. Y.; Xu, W. Q.; Zhao, B.; Ozaki, Y. Anal. Chem. 2008, 80, 3020.

19. Sun, L.; Yu, C.; Irudayaraj, J. Anal. Chem. 2008, 80, 3342.

20. Guo, S.; Wang, Y.; Wang, E. Nanotechnology 2007, 18, 405602.

21. Lee, S.; Kim, S.; Choo, J.; Shin, S. Y.; Lee, Y. H.; Choi, H. Y.; Ha, S.; Kang, K.; Oh, C. H. Anal. Chem. 2007, 79, 916.
22. Driskell, J. D.; Uhlenkamp, J. M.; Lipert, R. J.; Porter, M. D. Anal. Chem. 2007, 79, 4141.

23. Kim, J.-H.; Kim, J.-S.; Choi, H.; Lee, S.-M.; Jun, B.-H.; Yu, K.-N.; Kuk, E.; Kim, Y.-K.; Jeong, D. H.; Cho, M.-H.; Lee, Y.-S. Anal. Chem. 2006, 78, 6967.

24. Driskell, J. D.; Kwarta, K. M.; Lipert, R. J.; Porter, M. D.; Neill, J. D.; Ridpath, J. F. Anal. Chem. 2005, 77, 6147.

25. Lyandres, O.; Shah, N. C.; Yonzon, C. R.; Walsh, J. T., Jr.; Glucksberg, M. R.; Van Duyne, R. P. Anal. Chem. 2005, 77, 6134.

26. Grubisha, D. S.; Lipert, R. J.; Park, H.-Y.; Driskell, J.; Porter, M. D. Anal. Chem. 2003, 75, 5936.

27. Graham, D.; Mallinder, B. J.; Whitcombe, D.; Watson, N. D.; Smith, W. E. Anal. Chem. 2002, 74, 1069.

28. Cao, Y. C.; Jin, R.; Mirkin, C. A. Science 2002, 297, 1536.

29. Nie, S.; Emory, S. R. Science 1997, 275, 1102.

30. Ni, J.; Lipert, R. J.; Dawson, G. B.; Porter, M. D. Anal. Chem. 1999, 71, 4903.

31. Terry, L. A.; White, S. F.; Tigwell, L. J. J. Agric. Food Chem. 2005, $53,1309$.

32. Kanda, V.; Kariuki, J. K.; Harrison D. J.; Mcdermott, M. T. Anal. Chem. 2004, 76, 7252

33. Jaiswal, J. K.; Mattoussi, H.; Mauro J. M.; Simon, S. M. Nat. Biotechnol. 2003, 21, 47 .

34. Rowe, C. A.; Scruggs, S. B.; Feldstein, M. J.; Golden J. P.; Ligler, F. S. Anal. Chem. 1999, 71, 431.

35. Bruchez, M., Jr.; Moronne, M.; Gin, P.; Weiss, P.; Alivisatos, A. P. Science 1998, 281, 2013.

36. Chan W. C.; Nie, S. Science 1998, 281, 2016.

37. Zheng, Y.; Chen, H.; Liu, X.-P.; Jiang, J.-H.; Luo, Y.; Shen, G.-L.; Yu, R.-Q. Talanta 2008, 77, 809.

38. Kerman, K.; Endo, T.; Tsukamoto, M.; Chikae, M.; Takamura, Y.; Tamiya, E. Talanta 2007, 71, 1494.

39. Cao, C.; Kim, J.; Kim, B.; Chae, H.; Yoon, H.; Yang, S.; Sim, S. Biosens. Bioelectron. 2006, 21, 2106.

40. Fang, Y.; Björn, P.; Stefan, L.; Wolfgang, K. Anal. Chem. 2004, 76,6765 .

41. Fernández-Sánchez, C.; McNeil, C. J.; Rawson, K.; Nilsson, O. Anal. Chem. 2004, 76, 5649.

42. Acevedo, B.; Perera, Y.; Ruiz, M.; Rojas, G.; Benitez, J.; Ayala, M.; Gavilondo, J. Clin. Chim. Acta 2002, 317, 55.

43. Seto, Y.; Iba, T.; Abe, K. Luminescence 2001, 16, 285.

44. Soukka, T.; Paukkunen, J.; Härmä, H.; Lönnberg, S.; Lindroos, H.; Lövgren, T. Clin. Chim. 2001, 47, 1269.

45. Frens, G. Nat. Phys. Sci. 1973, 241, 20. 